\title{
O TEMPO E MANEIRA DE UTILIZAÇÃO DO CELULAR PODEM PREDISPOR À LESÕES MUSCULOESQUELÉTICAS: ESTUDO CASO-CONTROLE
}

\section{TIME AND CHARACTERISTICS OF CELL PHONE USAGE MAY PREDISPOSE MUSCULOSKELETAL INJURIES: CASE-CONTROL STUDY}

\author{
Luísa Cedin \\ Andréa Luciana Gomes Narcizo ${ }^{1}$ \\ Carolina Mitie Iwata ${ }^{2}$ \\ Amanda Antunes Henke Carrano ${ }^{2}$ \\ Danilo Harudy Kamonseki ${ }^{1}$
}

\section{RESUMO}

Objetivo: avaliar e comparar o tempo de utilização, postura e o manuseio do celular entre voluntários com e sem dor na região dos membros superiores ou da coluna cervical. Métodos: Este estudo possui um delineamento observacional transversal. Foram avaliados o tempo e finalidade, a maneira e postura de utilização do celular utilizando questionários com questões abertas de 24 participantes com dor na região da coluna cervical ou membro superior e 24 do grupo controle, que não apresentavam dor, com gênero e idade pareados entre os grupos. As comparações entre grupos foram realizadas por meio do Teste $\mathrm{T}$ de student para as variáveis contínuas e para as categóricas o Qui quadrado. Resultados: o grupo com dor apresentou maior utilização do celular sem apoio, tempo para digitar, internet e chamada do que o grupo controle $(p<0,05)$. Não houve diferença entre os grupos para a utilização da agenda, ouvir músicas e jogar no celular e tipo de manuseio $(p>0,05)$. Conclusão: $O$ tempo de utilização do celular para digitar, usar a internet $\mathrm{e}$ chamadas, a maneira de digitar e a postura sem apoio foram maiores em voluntários com dor, sugerindo que esses fatores podem influenciar no desenvolvimento de dor musculoesqueléticas na região de cervical e de membros superiores.

DESCRITORES: Telefones celulares. Doenças musculoesqueléticas. Fatores de risco.

\begin{abstract}
Objective: To evaluate and compare the time of cell phone use among volunteers with and without pain in the upper limbs and cervical spine area. Methods: This is an observational cross-sectional study. Questionnaires about cell phone usage were applied in 24 participants reporting pain in the cervical spine or upper limb and 24 participants without pain, aged 20 to 32 years old. The exclusion criteria were history of previous surgery, trauma and orthopedic injury or usage of upper limbs immobilization over the past six months. The comparisons between groups were performed using the $t$ student test for continuous data, and Chi-square test for categorical data. Results: The pain group showed greater use of cell phone in a sitting position and without any support, while the control group used it in a sitting position with support $(p<0.05)$. Cell phone usage time for typing messages, browsing the web and making phone calls were greater in the pain group than in the control group $(p<0.05)$. There was no difference between the groups for using the calendar, listening to music and playing games on mobile $(p>0.05)$. Conclusion: The time using the cell phone to type, use the internet and make calls, as well as the posture adopted and the usual handling of these devices are different between groups, suggesting that these factors may influence the musculoskeletal pain in the cervical region and upper limbs development.
\end{abstract}

DESCRIPTORS: Cell phones. Musculoskeletal diseases. Risk factors.

1: Docente. Instituto de Ciências da Saúde da Universidade Paulista - UNIP, Sorocaba, SP- Brasil.

2: Discente. Instituto de Ciências da Saúde da Universidade Paulista - UNIP, Sorocaba, SP- Brasil. 
s smartphones são amplamente utilizados por possuírem diversas funções, como enviar mensagens, navegar na internet, fazer ligações, ouvir músicas, assistir vídeos, tirar fotos e também podem ser utilizados para jogos ${ }^{1}$. Devido ao tempo excessivo e os movimentos repetitivos pela diversidade de recursos disponíveis, a utilização dos smartphones podem predispor ao surgimento de dor nos membros superiores e na região da coluna cervical ${ }^{2-4}$.Estudos prévios ${ }^{4-7}$ myofascial pain syndrome $(70.37 \%$ observaram associação entre a utilização do celular e tendinopatia do músculo extensor longo do polegar e de outros da região do punho, síndrome dolorosa miofascial, Síndrome de Quervain's e alterações degenerativas da primeira articulação carpometacarpal.

Durante a utilização do celular, outros fatores podem estar associados à etiologia de transtornos musculoesqueléticos, como, por exemplo, o teclado situado em pequeno espaço pode gerar maior tensão estática nos membros superiores ${ }^{2,8,9}$. Ainda, a posição que os usuários adquirem enquanto manuseiam seus aparelhos, como a coluna cervical em flexão, punhos e cotovelos em posição não neutra ${ }^{3,10,11}$, e outras posturas incorretas mantidas por longos períodos, no qual algumas pessoas podem considerar confortáveis, mas que podem gerar sobrecarga em diversas estruturas ${ }^{4,12}$.

Atualmente, poucas pesquisas investigaram as características de utilização que podem influenciar no desenvolvimento de dor musculoesquelética. Gustafssonet al. ${ }^{12}$ reali- zaram um estudo caso-controle para comparar a postura e modo de utilização entre pessoas com e sem dor musculoesqueléticos na região dos membros superiores, e observaram que os voluntários com sintomas apresentaram menor atividade do músculo abdutor longo do polegar, maior velocidade e menos pausa ao digitar. No entanto, devido à crescente utilização dos celulares, dos recursos disponíveis e de alterações no padrão de utilização, ainda são necessárias novas pesquisas para verificar outros possíveis fatores associados ao desenvolvimento de lesões musculoesqueléticas em usuários de celulares, como o tempo, postura e o manuseio do celular.

Neste contexto, o objetivo deste estudo foi avaliar e comparar o tempo de utilização, postura e o manuseio do celular entre voluntários com e sem dor na região dos membros superiores ou na coluna cervical.

\section{MÉTODOS}

Este estudo possui um delineamento observacional transversal. Participaram deste estudo 48 voluntários de ambos os gêneros, com e sem dor na região dos membros superiores ou coluna cervical. Para o recrutamento dos indivíduos foram divulgados anúncios pela universidade, clínicas da cidade, no rádio e meio digital. No Grupo Dor (GD), foram alocados 24 voluntários que possuíam dor nos membros superiores ou coluna cervical, e no Grupo Controle (GC), 24 indivíduos que não apresentavam dor, com pareamento do gênero e idade. 
Foram considerados como critérios de inclusão para ambos os grupos: utilização frequente do telefone celular e faixa etária entre 18 e 65 anos, para o GD os voluntários deveriam apresentar dor na região dos membros superiores ou coluna cervical, e para o GD, não apresentar dor nos membros superiores e na coluna cervical. Foram excluídos os voluntários que relataram histórico de cirurgia prévia, trauma e lesões ortopédicas ou utilização de imobilizações nos membros superiores nos últimos seis meses. Esta pesquisa foi aprovada pelo Comitê de Ética em Pesquisa com Seres Humanos da Universidade Paulista (CAAE: 53418716.6.0000.5512) e os voluntários que participaram do estudo assinaram o termo de consentimento livre e esclarecido.

\section{Procedimentos}

Foram coletadas informações sobre a idade (anos), gênero, a maneira como utilizam os celulares (com os polegares ou indicadores) e o tempo de utilização conforme a finalidade (minutos), como telefonemas, envio de mensagens, jogos, internet, agenda e músicas utilizando questionário com questões abertas.

Os voluntários do GD forneceram informações sobre nível de dor e os locais dos membros superiores e coluna cervical. Essas informações eram coletadas de forma verbal com perguntas realizadas diretamente aos voluntários. A dor foi mensurada por meio da Escala Numérica de Dor (END) de 11 pontos.
Essa escala possui pontuação de 0 (sem dor) à 10 (dor máxima). A confiabilidade teste-reteste foi verificada por estudo prévio e apresentou coeficiente de correlação intraclasse $(\mathrm{CCl})$ de 0,84, e diferença mínima clínica importante (MCID) de 15\% para dor crônica musculoesquelética ${ }^{13,14}$ the short form of the Disabilities of the Arm, Shoulder and Hand, the Patient-Specific Functional Scale, a numeric pain rating scale, and the Global Perceived Effect scale in patients with shoulder disorders. BACKGROUND Most instruments for shoulder disorders were developed in English. For their use in Brazil, translation into Brazilian Portuguese, cross-cultural adaptation, and testing of measurement properties of these instruments are required. METHODS The SPADI was translated and cross-culturally adapted according to current guidelines. Measurement properties of internal consistency, reproducibility, construct validity, ceiling and floor effects, and responsiveness of all instruments were tested in 100 patients with shoulder disorders. The patients filled out the questionnaires on 3 occasions: at baseline, 24 to 48 hours after baseline, and 4 weeks after baseline. RESULTS The instruments showed adequate internal consistency (Cronbach alpha between .78 and .93 .

\section{Análise estatística}

A análise dos dados foi realizada por meio do programa Statistica 12. As características contínuas dos voluntários foram 
apresentadas por meio de média e desvio padrão e as categóricas por frequência e porcentagem. As comparações entre GD e $\mathrm{GC}$ foram realizadas por meio do Teste $\mathrm{T}$ de student para as variáveis contínuas e para as categóricas o Qui quadrado. Odds ratio com $95 \%$ de Intervalo de confiança (95\% IC) foi calculado para verificar a presença e a força de associações. O nível de significância foi considerado $p<0,05$.

\section{RESULTADOS}

A idade, gênero e a dominância entre os membros superiores dos voluntários não apresentaram diferença estatística $(p>0,05)$ entre os grupos (Tabela 1).

As descrições dos locais e nível de dor dos membros dominante e não dominantes estão na tabela 2 . Vinte e três $(95,8 \%)$ dos voluntários relataram dor na região da coluna cervical, com média de dor de 7,3 $\pm 2,5$.

Com relação a principal postura adotada, o GD utilizou mais o celular sentado e sem apoio, e o GC sentado com apoio ( $p<$ $0,05)$. O manuseio do aparelho apresentou diferença significativa $(p<0,05)$, sendo que o GD utilizou mais o celular com ambos os polegares (Tabela 3).

O tempo de utilização do celular para digitar, internet e realizar chamadas foi maior no $G D$ do que no $G C(p<0,05)$. Não houve diferença entre os grupos para a utilização da agenda, ouvir músicas e jogar no celular $(p>0,05)$ (Tabela 4).

\section{DISCUSSÃO}

A crescente utilização do telefone celular pela população para diversas finalidades pode ser um fator de risco para o desenvolvimento de doenças, e os resultados deste estudo indicam que o tempo de utilização para digitar, utilizar internet e realizar chamadas, e a postura sem apoio podem influenciar o desenvolvimento de dor musculoesqueléticas.

Os resultados desse estudo indicam que o tempo de utilização do celular nos participantes que relataram sintomas álgicos foi maior em comparação com os que não possuem dor. Estes resultados corroboram com estudos prévios ${ }^{2,15}$ que observaram relação entre o tempo de utilização, alterações posturais e a dor musculoesquelética ${ }^{1}$. Berolo et al ${ }^{2}$ observaram associação do tempo total de utilização com dor na região dos ombros e coluna cervical, e o período jogando e utilizando a internet com os síntomas na região do polegar. Além disso, Jung et al. ${ }^{15}$ observaram influência do uso do celular sobre a postura da cabeça e escápula, e Lee et al. ${ }^{16}$ and to compare the effects of phone handling with one hand and with both hands. [Subjects] The study subjects were asymptomatic women 2022 years of age. [Methods] The subjects sat in a chair with their feet on the floor and the elbow flexed, holding a smartphone positioned on the thigh. Subsequently, the subjects typed the Korean anthem for 3 min, one-handed or with both hands. Each subject repeated the task three times, with a 5-min rest period between tasks to minimize fatigue. Electromyography 
Tabela 1. Características dos participantes

\begin{tabular}{l|c|c|c}
\hline \multicolumn{1}{c|}{ Variáveis } & Grupo com Dor & Grupo Controle & $p$-value \\
\hline Idade & $24,3 \pm 3,5$ & $24,3 \pm 3,5$ & 1,00 \\
$\quad$ anos & $14(58)$ & $14(58)$ & 1,00 \\
$\begin{array}{l}\text { Gênero } \\
\text { Feminino n (\%) }\end{array}$ & $20(87,5)$ & $22(91,7)$ & \\
Dominância & $3(12,5)$ & $2(8,3)$ & 0,63 \\
Direito, n (\%) & & \\
Esquerdo, $\mathrm{n}(\%)$ &
\end{tabular}

Tabela 2. Descrição dos locais e nível de dor do Grupo Dor

\begin{tabular}{l|c|c|c|c}
\hline \multirow{2}{*}{ Variáveis } & \multicolumn{2}{|c|}{ Membro dominante } & \multicolumn{2}{c}{ Membro não dominante } \\
\cline { 2 - 5 } & $\mathrm{n}(\%)$ & END & $\mathrm{n}(\%)$ & END \\
\hline Polegar & $19(79,1)$ & $5,2 \pm 2,3$ & $16(66,6)$ & $4,8 \pm 2,7$ \\
Outros dedos & $5(20,8)$ & $3,4 \pm 1,5$ & $5(20,8)$ & $3,4 \pm 1,5$ \\
Dorso da mão & $4(16,6)$ & $2,5 \pm 1,7$ & $3(12,5)$ & $1,6 \pm 1,1$ \\
Palma da mão & $3(12,5)$ & $4,6 \pm 1,1$ & $3(12,5)$ & $4,6 \pm 1,1$ \\
Cotovelo & $9(37,5)$ & $4,3 \pm 3,0$ & $9(37,5)$ & $4,7 \pm 2,9$ \\
Ombro & $16(66,6)$ & $5,6 \pm 2,5$ & $15(62,5)$ & $5,3 \pm 2,4$ \\
\hline
\end{tabular}

Abreviação: END, Escala Numérica de Dor. Valores contínuos expressos por média \pm desvio padrão, e os categóricos por frequência (\%).

Tabela 3. Comparação da postura e manuseio do aparelho entre os Grupo com dor e controle

\begin{tabular}{|c|c|c|c|c|}
\hline Principal Postura & $\begin{array}{c}\text { Grupo com } \\
\text { Dor }\end{array}$ & $\begin{array}{l}\text { Grupo } \\
\text { Controle }\end{array}$ & OR (95\%IC) & $p$-value \\
\hline Sentado sem apoio, n (\%) & $16(66,6)$ & $8(33,3)$ & $4,0(1,2-13,2)$ & 0.02 \\
\hline Sentado com apoio, n (\%) & $3(12,5)$ & $11(45,8)$ & $0,16(0,03-0,7)$ & 0.011 \\
\hline Em pé, n (\%) & $1(4,1)$ & $0(0)$ & $3,12(0,12-80,6)$ & 0,49 \\
\hline Deitado, n (\%) & $4(16,6)$ & $5(20,8)$ & $0,7(0,17-3,26)$ & 0,71 \\
\hline \multicolumn{5}{|l|}{ Manuseio do aparelho } \\
\hline Ambos os polegares, $\mathrm{n}(\%)$ & $20(83,3)$ & $12(50)$ & $5,0(1,3-19,0)$ & 0,01 \\
\hline Polegar direito, n (\%) & $3(12,5)$ & $8(33,3)$ & $0,28(0,06-1,25)$ & 0,09 \\
\hline Polegar esquerdo, n (\%) & $1(4,1)$ & $1(4,1)$ & $1,0(0,059-16,9)$ & 1,0 \\
\hline Indicador direito, n (\%) & $0(0)$ & $3(12,5)$ & $0,12(0,006-2,56)$ & 0,17 \\
\hline
\end{tabular}


Tabela 4. Comparação do tempo de utilização do celular entre os grupos Dor e Controle

\begin{tabular}{l|c|c|c}
\hline \multicolumn{1}{c|}{ Variáveis } & Grupo com Dor & Grupo Controle & $p$-value \\
\hline Digitar mensagens, min & $380,0^{*} \pm 153,9$ & $159,5 \pm 77,0$ & 0,001 \\
Internet, min & $322,5^{*} \pm 165,7$ & $133,7 \pm 69,0$ & 0,001 \\
Agenda, min. & $22,2 \pm 48,7$ & $8,9 \pm 11,2$ & 0,206 \\
Chamadas, min & $72,9^{*} \pm 74,6$ & $23,3 \pm 25,4$ & 0,003 \\
Ouvir música, min & $115,4 \pm 124,5$ & $62,9 \pm 50,2$ & 0,061 \\
Jogar no celular, min & $48,7 \pm 57,8$ & $32,5 \pm 64,3$ & 0,362 \\
\hline
\end{tabular}

Valores contínuos expressos por média \pm desvio padrão. * $p<0,05$

(EMG sobre a fadiga muscular do trapézio superior .Os resultados desse estudo podem auxiliar no desenvolvimento de programas de conscientização e prevenção de lesões ,abordando os efeitos que a utilização do celular em excesso pode gerar no desenvolvimento de lesões. Sendo assim, estudos futuros são necessários para verificar os efeitos de programas de prevenção e alterações no padrão de utilização do celular sobre as lesões musculoesqueléticas.

Os voluntários com dor utilizaram mais o celular sem apoio em comparação com os que não possuíam dor. De acordo com os resultados desse estudo, utilizar o celular sem apoio pode aumentar três vezes (OR 4, 95\% IC 1,2-13,2) a chance de ter dor musculoesquelética na região dos membros superiores. Isso sugere que o apoio para utilizar o celular pode auxiliar na manutenção da postura mais adequada, prevenindo alterações que podem predispor às desordens musculoesqueléticas na região do pescoço, ombros, punhos e dedos, devido a contração isométrica prolongada dos músculos destas regiões ${ }^{17-20}$ Shoulder, and
Hand Questionnaire (QuickDASH. Além do apoio durante a utilização do celular, outros fatores também influenciam no desenvolvimento de lesões, como o descrito por Gold et al. ${ }^{10}$, que os indivíduos permanecem por longas horas em posturas inadequadas, com o pescoço flexionado, braços suspensos, cotovelos com ângulo menores que $90^{\circ}$, punho em posição não neutra e realizam movimentos repetitivos em múltiplas direções com o polegar. Segundo Park et al. ${ }^{19}$ there has been a rapid increase in the use of mobile phones and other small hand-held devices for communication. A forward head posture (turtle neck posture, a flexão excessiva da cabeça, como observado por Gold et al. ${ }^{10}$ pode produzir tensões e alteração na curvatura da coluna cervical.

Além disso, os resultados desse estudo sugerem que a digitação dos celulares com ambos os polegares pode aumentar quatro vezes (OR 5,0, 95\%IC1,3 - 19,0) chance de dor nos membros superiores. Lee et al. ${ }^{16}$ and to compare the effects of phone handling with one hand and with both hands. [Subjects] The study subjects were asymptomatic women 20 22 years of age. [Methods] The subjects sat in 
a chair with their feet on the floor and the elbow flexed, holding a smartphone positioned on the thigh. Subsequently, the subjects typed the Korean anthem for $3 \mathrm{~min}$, one-handed or with both hands. Each subject repeated the task three times, with a 5-min rest period between tasks to minimize fatigue. Electromyography (EMG observaram que utilizar o celular com uma mão há maior dor pressórica e atividade do músculo trapézio superior em comparação com as duas mãos. Sendo assim, postura correta e a utilização do celular com apoio são informações importantes que podem ser incluídas nos programas de prevenção ${ }^{21,22}$. Mas ainda há necessidade de estudos para verificar os efeitos dessas intervenções.

Este estudo apresentou algumas limitações. O avaliador não era cegado para os grupos avaliados e não houve coleta de dados sobre a ocupação dos participantes, o que pode influenciar o resultado do estudo. Estudos futuros são necessários para verificar os efeitos da alteração no tempo e postura de utilização sobre a dor e função de voluntários com dor musculoesquelética associada à utilização em excesso do celular.

\section{CONCLUSÃO}

Os voluntários que relataram dor na região da coluna cervical ou membros superiores apresentaram maior tempo de utilização do celular e postura sem apoio em comparação com o grupo controle, indicando que esses fatores podem estar relacionados ao desenvolvimento de desordens musculoesqueléticas.

\section{REFERÊNCIAS}

1. Park N, Kim YC, Shon HY, Shim H. Factors influencing smartphone use and dependency in South Korea. Comput Human Behav. 2013; 29(4):1763-1770.

2. Berolo S, Wells RP, Amick BC. Musculoskeletal symptoms among mobile hand-held device users and their relationship to device use: A preliminary study in a Canadian university population. Appl Ergon. 2011; 42(2):371-378.

3. Kietrys DM, Gerg MJ, Dropkin J, Gold JE. Mobile input device type, texting style and screen size influence upper extremity and trapezius muscle activity, and cervical posture while texting. Appl Ergon. 2015; 50:98-104.

4. Eapen C, Kumar B, Bhat AK, Venugopal A. Extensor Pollicis Longus Injury in Addition to De Quervain's with Text Messaging on Mobile Phones. J Clin Diagn Res. 2014; $8(11): 1-4$

5. Sharan D, Ajeesh PS. Risk factors and clinical features of text message injuries. Work 2012; 41:1145-1148.

6. Abdulla SY, Southerst D, Côté P, Shearer HM, Sutton D, Randhawa K, et al. Is exercise effective for the management of subacromial impingement syndrome and other soft tissue injuries of the shoulder? A systematic review by the Ontario Protocol for Traffic Injury Management (OPTIMa) Collaboration. Man Ther. 2015; 20(5):646-656.

7. Ming Z, Pietikainen S, Hänninen O. Excessive texting in pathophysiology of first carpometacarpal joint arthritis. Pathophysiology. 2006; 13(4):269-270.

8. Xiong J, Muraki S. Effects of age, thumb length and screen size on thumb movement coverage on smartphone touchscreens. Int J Ind Ergon. 2016; 53:140-8. 
9. Darowish M, Sharma J. Evaluation and treatment of chronic hand conditions. Med Clin North Am. 2014; 98(4):801-15.

10. Gold JE, Driban JB, Thomas N, Chakravarty T, Channell $\mathrm{V}$, Komaroff E. Postures, typing strategies, and gender differences in mobile device usage: An observational study. Appl Ergon. 2012; 43(2):408-412.

11. Lin HT, Kuo LC, Liu HY, Wu WL, Su FC. The three-dimensional analysis of three thumb joints coordination in activities of daily living. Clin Biomech. 2011; 26(4):371-376.

12. Gustafsson E, Johnson PW, Hagberg M. Thumb postures and physical loads during mobile phone use - a comparison of young adults with and without musculoskeletal symptoms. J Electromyogr Kinesiol. 2010; 20(1):127-135.

13. Puga VO de O, Lopes AD, Shiwa SR, Alouche SR, Costa LOP. Clinimetric testing supports the use of 5 questionnaires adapted into Brazilian Portuguese for patients with shoulder disorders. J Orthop Sports Phys Ther. 2013; 43(6):404-413.

14. Salaffi F, Stancati A, Silvestri CA, Ciapetti A, Grassi W. Minimal clinically important changes in chronic musculoskeletal pain intensity measured on a numerical rating scale. Eur J Pain. 2004; 8(4):283-91.

15. Jung SI, Lee NK, Kang KW, Kim K, Lee DY. The effect of smartphone usage time on posture and respiratory function. J Phys Ther Sci. 2016; 28(1):186-9.

16. Lee M, Hong Y, Lee S, Won J, Yang J, Park S, et al. The effects of smartphone use on upper extremity muscle activity and pain threshold. J Phys Ther Sci. 2015; 27(6):1743-5.
17. Mintken PE, Glynn P, Cleland JA. Psychometric properties of the shortened disabilities of the Arm, Shoulder, and Hand Questionnaire (QuickDASH) and Numeric Pain Rating Scale in patients with shoulder pain. J Shoulder Elbow Surg. 2009; 18(6):920-6.

18. Li ZM, Tang J. Coordination of thumb joints during opposition. J Biomech. 2007; 40(3):502-10.

19. Park J, Kim K, Kim N, Choi I, Lee S. A Comparison of Cervical Flexion , Pain , and Clinical Depression in Frequency of Smartphone Use. Int J Bio-Science Bio-Technology. 2015; 7(3): 183-90.

20. Lee $S$, Lee $D$, Park J. Effect of the cervical flexion angle during smart phone use on muscle fatigue of the cervical erector spinae and upper trapezius. J Phys Ther Sci. 2015; 27(6):1847-9.

21. Gustafsson E. Ergonomic recommendations when texting on mobile phones. Work. 2012; 41:5705-6.

22. Gustafsson $E$, Johnson $P W$, Lindegård $A$, Hagberg $M$. Technique, muscle activity and kinematic differences in young adults texting on mobile phones. Ergonomics. 2011; 54(5): 477-87.

\section{CORRESPONDÊNCIA}

Danilo Harudy Kamonseki

Avenida independência, 1230, Édens, Sorocaba-SP.

E-mail: fisiot.danilo@hotmail.com 